Hautarzt 2022 $\cdot 73: 234-235$

https://doi.org/10.1007/s00105-022-04963-0 Angenommen: 2. Februar 2022

(C) The Author(s), under exclusive licence to Springer Medizin Verlag GmbH, ein Teil von Springer Nature 2022

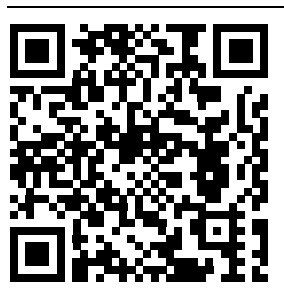

QR-Code scannen \&Beitrag online lesen

\title{
NIM: Neue Ideen für die Medizin
}

\author{
Eine neue Rubrik in der Zeitschrift Der Hautarzt
}

\author{
Alexander Zink ${ }^{1,2} \cdot$ Natalia Kirsten $^{3}$ \\ ${ }^{1}$ Klinik und Poliklinik für Dermatologie und Allergologie, Fakultät für Medizin, Technische Universität \\ München, München, Deutschland \\ ${ }^{2}$ Division of Dermatology and Venereology, Department of Medicine Solna, Karolinska Institutet, \\ Stockholm, Schweden \\ ${ }^{3}$ Institut für Versorgungsforschung in der Dermatologie und bei Pflegeberufen (IVDP), \\ Universitätsklinikum Hamburg-Eppendorf (UKE), Hamburg, Deutschland
}

Liebe Kolleginnen und Kollegen,

die Digitalisierung wird auch die Medizin stark verändern. Bereits jetzt ergeben sich zahlreiche neue Fragestellungen, $v$. a. aber auch neue Möglichkeiten, die wir für die Dermatologie, Allergologie und Venerologie frühzeitig und proaktiv nutzen sollten.

Dazu gehören beispielsweise der Einsatz von Apps und künstlicher Intelligenz zur Evaluation von Befunden [1, 2], Wearables und Smart Skin zur Messung verschiedener Körperfunktionen [3], Chatbots, Netzwerkanalysen und 3-DGanzkörpervermessung [4] sowie vieles weitere mehr. Für einen Überblick sowie eine Präsentation einer gezielten Auswahl digitaler Technologien und neuer Ideen, die für die Dermatologie, Venerologie und Allergologie sowie angrenzende Fachrichtungen und die Medizin relevant erscheinen, wurde die Rubrik "NIM: Neue Ideen für die Medizin“ geschaffen.

So sollen verschiedenste innovative Technologien und digitale Lösungen, die innerhalb der Dermatologie zum Einsatz kommen können, in der neuen Rubrik detailliert und praxisnah dargestellt werden. Gleichzeitig werden aber auch in anderen Disziplinen fest etablierte Ideen und Innovationen vorgestellt werden, die für die Medizin interessant sein könnten. Hierzu zählen z.B. die partizipativen (mitarbeitereinbeziehenden) Optimierungssysteme zur Nutzung des Ideenpotenzials der Vielzahl unterschiedlicher Mitarbeiter innerhalb der Automobilindustrie oder der Einsatz von "optical character recognition" (OCR) zur automatisierten
Zuordnung und Klassifizierung großer Mengen an Verträgen und Dokumenten in der Rechtswissenschaft.

Eine Zielvorstellung sowie der Anspruch an die Artikel sind dabei immer, digitale Lösungsansätze aufzuzeigen, um nützliche, kreative oder unterhaltsame Lösungen für gegebene dermatologische Probleme zu finden.

An der Fakultät für Medizin der TU München organisiert die Klinik und Poliklinik für Dermatologie und Allergologie auch unter Mitwirken des Arbeitskreises Digitale Dermatologie der Deutschen Dermatologischen Gesellschaft seit 2019 2-mal jährlich ein Symposium „New Ideas for Medicine (NIM) - Digitale Kompetenz für Mediziner" (๑ Abb. 1) mit Teilnehmern und Referenten aus vielen Teilen der Welt. Die ersten NIM-Artikel basieren auf Vorträgen des Symposiums, so auch der Beitrag in diesem Heft von Peter Soyer und Kollegen aus Australien [4].

Machen Sie mit und berichten Sie von innovativen Ideen und Lösungen für die Medizin.

Ihre

Natalia Kirsten und Alexander Zink

\section{Korrespondenzadresse}

PD Dr. Dr. med. Alexander Zink

Klinik und Poliklinik für Dermatologie und Allergologie, Fakultät für Medizin, Technische Universität München

Biedersteiner Str. 29, 80802 München,

Deutschland

alexander.zink@tum.de 


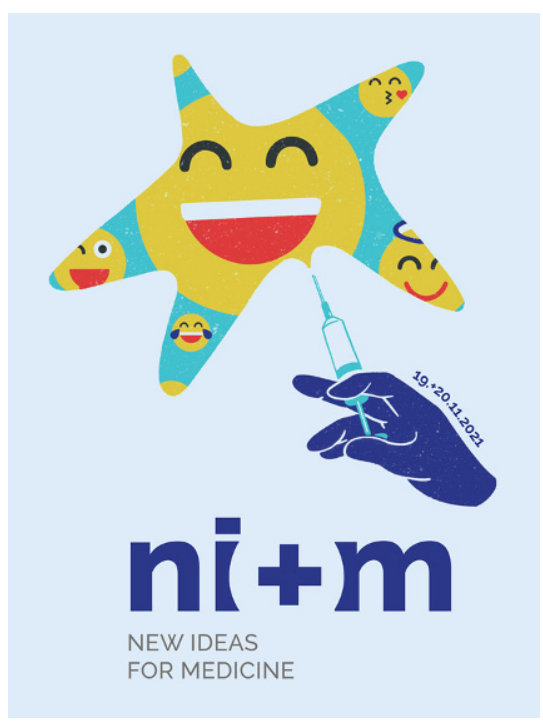

Abb. $1 \Delta$ Logo des NIM-Symposiums im November 2021 (@ Felix King, mit freundl. Genehmigung)

Interessenkonflikt. A. Zink und N. Kirsten geben an dass kein Interessenkonflikt besteht.

\section{Literatur}

1. Winkler JK, SiesK, FinkC, TobererF, EnkA, Abassi MS, Fuchs T, Blum A, Stolz W, Coras-Stepanek B, Cipic R, Guther S, Haenssle HA (2021) Kollektive menschliche Intelligenz übertrifft künstliche Intelligenz in einem Quiz zur Klassifizierung von Hautläsionen. JDtsch Dermatol Ges 19(8):1178-1185

2. Becker M (2022) Swarm learning for decentralized healthcare. Hautarzt. https://doi.org/10.1007/ s00105-021-04940-z

3. Kiani C, Kain A, Zink A (2022) Wearables and smart skin as new tools for clinical practice and research in dermatology. JEADV Clin Pract. https://doi.org/ $10.1002 /$ jvc 2.2

4. Rutjes C, Torrano J, Soyer HP (2022) A 3D total-body photography research network: the Australian experiment. Hautarzt. https://doi.org/10.1007/ s00105-021-04938-7

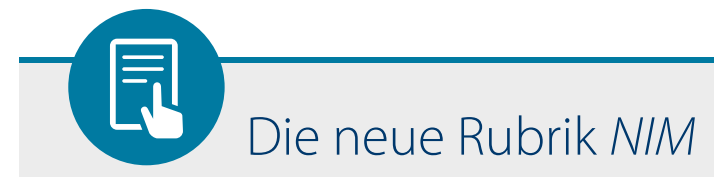

Ab sofort finden Sie in der Rubrik „Neue Ideen für die Medizin“ (NIM) Artikel zu innovativen digitalen Technologien, die in der Dermatologie eingesetzt werden.

Freuen Sie sich 2022 auf folgende, von nationalen und internationalen Expert*innen verfasste Kurzbeiträge:

- A 3D total-body photography research network: the Australian experiment (P. Soyer et al.)

- Swarm learning for decentralized healthcare (M. Becker)

- The role of dermatologists in social media: exploring the benefits and risks (F. Ahmed, J.B. Lipoff)

- Innovative eCME courses - an approach with tangible augmented reality (K. Böhm)

- 3D-Ganzkörperhautscanner (S. Sitaru et al.)

- Werden ärztliche Entscheidungen in Zukunft von der Künstlichen Intelligenz übernommen? (J. Welzel)

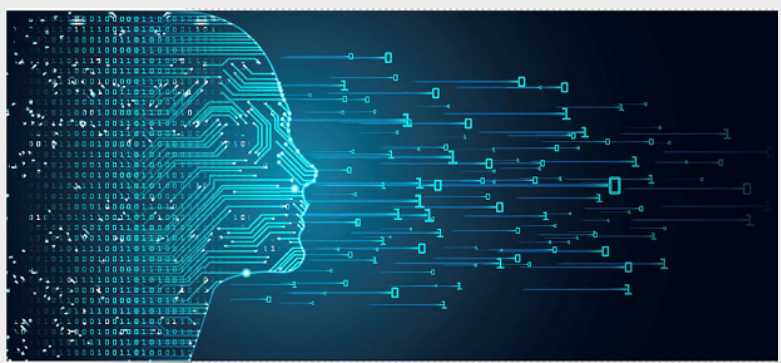

○ Ryzhi / Getty Images / iStock 